\title{
Formulation and evaluation of a topical niosomal gel containing a combination of benzoyl peroxide and tretinoin for antiacne activity
}

This article was published in the following Dove Press journal: International Journal of Nanomedicine

24 December 2014

Number of times this article has been viewed

\author{
Ankush Gupta',* \\ Sima Singh ${ }^{1, *}$ \\ Niranjan G Kotla' \\ Thomas J Webster ${ }^{2,3}$ \\ 'Department of Pharmaceutical \\ Sciences, Lovely Professional \\ University, Phagwara, Punjab, India; \\ ${ }^{2}$ Department of Chemical Engineering, \\ Northeastern University, Boston, \\ MA, USA; ${ }^{3}$ Center of Excellence \\ for Advanced Materials Research, \\ King Abdulaziz University, Jeddah, \\ Saudi Arabia \\ *These authors contributed equally \\ to this work
}

\begin{abstract}
A skin disease, like acne, is very common and normally happens to everyone at least once in their lifetime. The structure of the stratum corneum is often compared with a brick wall, with corneocytes surrounded by the mortar of the intercellular lipid lamellae. One of the best options for successful drug delivery to the affected area of skin is the use of elastic vesicles (niosomes) which can be transported through the skin through channel-like structures. In this study, a combination of tretinoin (keratolytic agent) and benzoyl peroxide (BPO) (a potent antibacterial) was given by using niosomes as promising carriers for the effective treatment of acne by acting on a pathogenic site. In this section, niosomal gel formulation encapsulated drugs have been evaluated for in vitro, ex vivo, and in vivo, for their predetermined characteristics; and finally the stability of the niosome gel was tested at different temperature conditions for understanding of the storage conditions required for maintaining the quality of formulation attributes. The prepared niosome was found to be in the range of $531 \mathrm{~nm}$ with a zeta potential of $-43 \mathrm{mV}$; the entrapment efficiencies of tretinoin (TRA) and BPO niosomes were found to be $96.25 \% \pm 0.56 \%$ and $98.75 \% \pm 1.25 \%$, respectively. The permeated amount of TRA and BPO from the niosomal gel after 24 hours was calculated as $6.25 \pm 0.14 \mu \mathrm{g} / \mathrm{cm}^{2}$ and $5.04 \pm 0.014 \mu \mathrm{g} / \mathrm{cm}^{2}$, respectively. A comparative drug retention study in Wistar rat skin using cream, an alcoholic solution, and a niosomal gel showed $11.54 \mu \mathrm{g}, 2.68 \mu \mathrm{g}$, and $15.54 \mu \mathrm{g}$ amounts of TRA and $68.85 \mu \mathrm{g}, 59.98 \mu \mathrm{g}$, and $143.78 \mu \mathrm{g}$ amounts of BPO were retained in the layers of skin, respectively. In vivo studies of the niosomal gel and antiacne cream of TRA and BPO showed that the niosomal gel was more efficacious than the antiacne cream because niosomal gels with a 4.16-fold lower dose of BPO provided the same therapeutic index at targeted sites in comparison to the antiacne cream.
\end{abstract}

Keywords: antiacne combination therapy, rabbit ear pinna model, retention efficiency, therapeutic index

\section{Introduction}

The optimization of drug delivery through human skin is important in modern therapy. Clearly, the topical route of drug delivery for treating skin diseases offers an attractive alternative to the conventional drug-delivery methods of oral administration/injection, and it is becoming a most innovative research area in drug delivery. A skin disease like acne, is very common and normally happens to everyone once in their lifetime.

Acne vulgaris is a chronic inflammatory dermatosis which is notable for open and/or closed comedones (blackheads and whiteheads), and inflammatory lesions including papules, pustules, or nodules. It is a disorder of sebaceous follicles which are special pilosebaceous units located on the face, chest, and back. ${ }^{1}$ Propionibacterium acnes and Staphylococcus epidermidis have been recognized as pus-forming bacteria triggering inflammation in acne. ${ }^{2}$

The organism produces extracellular lipases that hydrolyze sebum triglycerides to glycerol and free fatty acids that have proinflammatory properties. ${ }^{3}$ The topical treatment of acne
Correspondence: Thomas J Webster Department of Chemical Engineering, Northeastern University, 360 Huntington Ave, Boston, MA 02115, USA

Tel +I 6173736585

Email th.webster@neu.edu 
includes topical retinoids, ${ }^{4,5}$ benzoyl peroxide (BPO), ${ }^{4,6}$ azelaic acid, ${ }^{7}$ erythromycin, ${ }^{8}$ clindamycin,${ }^{8}$ and combination therapies. ${ }^{9}, 10$ The adverse effects of topical antiacne agents include burning, erythema, scaling, flare-up, photosensitivity, and bacterial resistance. ${ }^{4}$ Tretinoin (TRA) and BPO are used individually and in a cyclic manner for acne treatment. Various conventional topical medicines are available in the market for treatment but have a less-therapeutic effect due to the efficient barrier properties of skin membranes. The structure of the stratum corneum is often compared with a brick wall made of corneocytes and surrounded by the mortar of the intercellular lipid lamellae. ${ }^{11}$ The best alternative for successful drug delivery to an affected area of skin is elastic vesicles (niosomes) which can be transported through the skin via channel-like structures. Moreover, they are too small - in the nanometer size range - to be detected by the immune system; furthermore, they can deliver the drug to the target site using lower drug doses in order to reduce side effects often experienced by topical routes by passing the complexity of the skin structure. ${ }^{12}$ The main advantages of using nanocarriers arise from their peculiar features, such as their tiny size, high surface energy, high surface area, composition, and architecture. ${ }^{12}$

Colloidal particulate carriers (including niosomes and liposomes) can act as drug reservoirs. ${ }^{13}$ Niosomes are unilamellar or multilamellar nonionic surfactant vesicles formed from synthetic nonionic surfactants by hydration, offering an alternative to liposomes. Niosomes are advantageous from a technical point of view as they possess greater stability and avoid some disadvantages associated with liposomes such as variable purity of phospholipids and high cost. ${ }^{14}$ These particulate carriers have been extensively studied as drug carriers in topical drug delivery. These carriers are advantageous because they increase drug stability, enhance therapeutic effects, prolong circulation time in a biological environment, and promote the uptake of the entrapped drugs into the target site while drug toxicity is diminished due to a reduction in nonspecific tissue uptake. ${ }^{15}$ Niosomes are capable of encapsulating both hydrophilic and lipophilic drugs and can serve as effective drug carriers. ${ }^{16}$

The vesicles serve as a soluble matrix and also serve as a local depot for sustained drug release; permeation enhancers of dermally active compounds; or a rate-limiting membrane barrier for the modulation of systemic absorption of drugs via dermal drug delivery. Here, Span 60 and cholesterol were selected as components of niosomes with BPO and tretinoin as model drugs for niosomal formulation. An in vitro permeation and retention study of BPO and tretinoin from niosomal gels were performed. Comparative antiacne activity of cream and niosomal gels was further evaluated by using a rabbit ear pinna model and we evaluated the impact of the niosome vesicle in drug delivery at the targeted site. ${ }^{17,18}$

\section{Material and methods Materials}

Tretinoin was procured ex gratis from Shalaks Pharmaceutical, New Delhi, India, while BPO was gifted by the H.K. Group, Mumbai, India. Span 60 and oleic acid were obtained from SD Fine Chemicals Limited, Mumbai, India, and cholesterol was obtained from Central Drug House (P) Ltd, New Delhi, India. Isopropanol was obtained from the Central Drug House (P) Ltd, carbopol 934 from Hi-media Laboratories PVT Ltd, Mumbai, India, and phosphotungstic acid from the Central Drug House (P) Ltd. All other materials and chemicals were of analytical grade.

\section{Incompatibility studies between drugs}

Incompatibility studies between drugs were performed using a stability chamber. In this study, equal amounts of each drug were taken, mixed uniformly, transferred to light resistant glass vials, and placed in a stability chamber. Individually, each drug was also placed at $65 \%$ relative humidity and $45^{\circ} \mathrm{C}$ temperature for 1 month. Infrared and ultraviolet (UV) spectroscopy were used to investigate any interaction between the drugs (Shimadzu Corporation, Kyoto, Japan, and Systronic, Gujrat, India; Model 2201, respectively).

\section{Susceptibility testing of BPO against S. epidermidis}

Susceptibility testing of S. epidermidis against BPO was checked by the disc diffusion method. S. epidermidis was incubated in a nutrient agar medium for 24 hours at $37^{\circ} \mathrm{C}$ and adjusted to yield approximately $1.0 \times 10^{8}$ colony forming units $/ \mathrm{mL}$. The prepared inoculums were added to molten agar, mixed, poured over the surface of the agar base, and left to solidify. A sterile paper disc was impregnated with test material and the disc was placed on the agar plates. The plates were incubated at $37^{\circ} \mathrm{C}$ for 24 hours under aerobic conditions. All disc diffusion tests were performed in three separate experiments and the antibacterial activity was expressed as the mean of the inhibition diameters (mm).

\section{Minimum inhibitory concentration of BPO for S. epidermidis}

The minimum inhibitory concentration (MIC) value was determined by the microdilution or broth dilution method. The calculated amount of broth was measured in a $10-\mathrm{mL}$ test tube and the glass test tube was sterilized by autoclaving at $121^{\circ} \mathrm{C}$ for 15 minutes. The medium was cooled and inoculated with $100 \mu \mathrm{L}$ of a bacterial suspension containing $10^{8}$ cells $/ \mathrm{mL}$. Then, various concentrations of $\mathrm{BPO}$ were added to respective test tubes which were incubated at $37^{\circ} \mathrm{C} \pm 1^{\circ} \mathrm{C}$ for 24 hours aerobically, and the 
growth of $S$. epidermidis was measured as function of turbidity at $660 \mathrm{~nm}$ using a UV spectrophotometer (Systronic 2201). ${ }^{19}$

\section{Preparation of niosomes}

An accurately weighed nonionic surfactant (Span 60) and cholesterol were dissolved in a chloroform:methanol (2:1) mixture and placed into a round bottom flask. The required quantity of stock solution of tretinoin $(4 \mathrm{mg} / \mathrm{mL})$ and BPO $(15 \mathrm{mg} / \mathrm{mL})$ were added in an optimized surfactant:cholesterol ratio as per batch size, then the organic solvent was removed by applying a vacuum. The temperature of the bath was set at $60^{\circ} \mathrm{C}$ and the flask was rotated at $160 \mathrm{rpm}$ until a smooth film was formed. Film was removed from the round bottom flask using a rotary evaporator equipment and put aside for 12 hours to remove traces of an organic solvent. Then, hydration of the film was performed with an optimized volume of water and saline, (in present study, water is used as hydration media for preparation of BPO niosomes, and saline is used for preparation of tretinoin niosomes), at above the lipidtransition temperature of the surfactant. Niosomes were formed and observed under a microscope (Tables 1 and 2). ${ }^{20}$

\section{Characterization of niosomes}

Morphological analysis by transmission electron microscopy

A drop of diluted niosome dispersion was applied to a carboncoated 300-mesh copper grid and was left for 1 minute to allow for some of the niosomes to adhere to the carbon substrate and be stained with $1 \%$ phosphotungstic acid. The remaining dispersion was removed by absorbing the drop with the corner of a piece of filter paper. Then, samples were examined and photographed with a Hitachi Ltd. (Tokyo, Japan) transmission electron microscope at $100 \mathrm{KV} .{ }^{21}$

\section{Particle size analysis by photon correlation} spectroscopy (dynamic laser light scattering [DLLS]) The vesicle sizes of niosomes were determined by light scattering based on laser diffraction using a Malvern Mastersizer (Model S, Ver. 2.15; Malvern Instruments, Malvern, UK). The apparatus consisted of a $\mathrm{HeNe}$ laser $(5 \mathrm{~mW})$ and a small-volume sample-holding cell. The sample was stirred using a magnetic stirrer bead to keep and maintain the sample in suspension.

\section{Zeta potential}

The significance of zeta potential is that its value can be related to the stability of colloidal dispersions. The zeta potential indicates the degree of repulsion between adjacent, similarly charged particles in dispersion. The zeta potential for the niosomal dispersion was determined using Malvern instruments. ${ }^{22}$

\section{Encapsulation efficiency}

Niosome-entrapped BPO and tretinoin could be separated from the free drug by the dialysis method. After hydration step, suspension of niosomes will form, which was filled in dialysis bags for removal of free drug present in suspension (MWCO-14000) and the free drugs were dialyzed for 24 hours into $100 \mathrm{~mL}$ of a phosphate buffer saline solution ( $\mathrm{pH}$ 7.4). After 24 hours, the dialysis niosomal suspension contained only the entrapped drug. From this niosomal suspension, $0.5 \mathrm{~mL}$ was taken and isopropanol was added up to $5 \mathrm{~mL}$, then the volume was increased to $10 \mathrm{~mL}$ with the respective solvent (tretinoin niosomal suspension with methanol and BPO suspension with ethanol); then, the absorbance of the resulting solution was measured at $234.8 \mathrm{~nm}$ and $348.6 \mathrm{~nm}$ for BPO and tretinoin, respectively. ${ }^{23}$

\section{Differential scanning calorimetry analysis}

for determining the phase transition temperature of niosomes (glass transition temperature)

Differential scanning calorimetry (DSC) experiments were performed with a differential scanning calorimeter (Shimadzu Corporation; model TA-50 WSI) calibrated with indium. Samples of multilamellar niosomes composed of Span 60:cholesterol (207:52 or 138:52 mg ratio) were submitted for DSC analysis. The analysis was performed on $40-\mu \mathrm{L}$ samples sealed in standard aluminum pans. Thermograms were obtained at a scanning rate of $10^{\circ} \mathrm{C} /$ minute. Zero point nine percent saline was employed as a reference. Samples were scanned between $30^{\circ} \mathrm{C}$ and $300^{\circ} \mathrm{C}$. The maximal excess heat capacity was defined as the phase transition temperature. ${ }^{24,25}$

Table I Ratio of surfactant and cholesterol used for niosome preparation, percent encapsulation of benzoyl peroxide

\begin{tabular}{|c|c|c|c|}
\hline $\begin{array}{l}\text { Serial } \\
\text { number }\end{array}$ & Niosomal formulation loaded with benzoyl peroxide & Hydration media & $\begin{array}{l}\text { \% encapsulation } \\
\text { efficiency (SD) }\end{array}$ \\
\hline $\mathrm{I}$ & Span 60:CH (69:35) weight $(\mathrm{mg})$ ratio & Water & $52.6 \% \pm 0.45 \%$ \\
\hline 2 & Span 60:CH (69:35) weight $(\mathrm{mg})$ ratio & Saline & $51.1 \% \pm 0.34 \%$ \\
\hline 3 & Span 60: $\mathrm{CH}$ (138:52) weight $(\mathrm{mg})$ ratio & Water & $98.75 \% \pm 1.25 \%$ \\
\hline 4 & Span 60:CH (I38:52) weight $(\mathrm{mg})$ ratio & Saline & $94.86 \% \pm 0.56 \%$ \\
\hline 5 & Span 60:CH (207:52) weight $(\mathrm{mg})$ ratio & Water & $92.4 \% \pm 0.49 \%$ \\
\hline 6 & Span 60:CH (207:52) weight $(\mathrm{mg})$ ratio & Saline & $89.06 \% \pm 0.76 \%$ \\
\hline
\end{tabular}

Notes: Stock solution of benzoyl peroxide was $15 \mathrm{mg} / \mathrm{mL}$. Percent encapsulation efficiency is the mean from triplicate experiments.

Abbreviations: $\mathrm{CH}$, cholesterol; $\mathrm{SD}$, standard deviation. 
Table 2 Ratio of surfactant and cholesterol used for niosome preparation, percent encapsulation of tretinoin

\begin{tabular}{|c|c|c|c|}
\hline $\begin{array}{l}\text { Serial } \\
\text { number }\end{array}$ & Niosomal formulation loaded with tretinoin & Hydration media & $\begin{array}{l}\text { \% encapsulation } \\
\text { efficiency (SD) }\end{array}$ \\
\hline 1 & Span 60: $\mathrm{CH}(69: 35)$ weight $(\mathrm{mg})$ ratio & Water & $24.5 \% \pm 0.65 \%$ \\
\hline 2 & Span $60: \mathrm{CH}(69: 35)$ weight $(\mathrm{mg})$ ratio & Saline & $43.25 \% \pm 0.35 \%$ \\
\hline 3 & Span 60: $\mathrm{CH}(138: 35)$ weight $(\mathrm{mg})$ ratio & Water & $48.25 \% \pm 0.82 \%$ \\
\hline 4 & Span 60: $\mathrm{CH}(138: 35)$ weight $(\mathrm{mg})$ ratio & Saline & $74.00 \% \pm 0.72 \%$ \\
\hline 5 & Span 60: $\mathrm{CH}(138: 52)$ weight $(\mathrm{mg})$ ratio & Water & $52.05 \% \pm 0.85 \%$ \\
\hline 6 & Span 60: $\mathrm{CH}(138: 52)$ weight $(\mathrm{mg})$ ratio & Saline & $86.45 \% \pm 0.54 \%$ \\
\hline 7 & Span 60:CH (207:52) weight $(\mathrm{mg})$ ratio & Water & $74.75 \% \pm 0.34 \%$ \\
\hline 8 & Span 60:CH (207:52) weight $(\mathrm{mg})$ ratio & Saline & $96.25 \% \pm 0.56 \%$ \\
\hline 9 & Span 60: $\mathrm{CH}(276: 52)$ weight $(\mathrm{mg})$ ratio & Water & $61.25 \% \pm 0.63 \%$ \\
\hline 10 & Span 60: $\mathrm{CH}(276: 52)$ weight $(\mathrm{mg})$ ratio & Saline & $70.75 \% \pm 0.43 \%$ \\
\hline
\end{tabular}

Notes: Stock solution of tretinoin $4 \mathrm{mg} / \mathrm{mL}$. Percent encapsulation efficiency is the mean from triplicate experiments.

Abbreviations: $\mathrm{CH}$, cholesterol; SD, standard deviation.

\section{Stability studies of the niosomal formulation}

The ability of vesicles to retain the drug was assessed by keeping the niosomal gel at three different temperature conditions, ie, refrigeration temperature $\left(4^{\circ} \mathrm{C}-8^{\circ} \mathrm{C}\right)$, room temperature $\left(25^{\circ} \mathrm{C} \pm 2^{\circ} \mathrm{C}\right)$, and oven temperature $\left(45^{\circ} \mathrm{C} \pm 2^{\circ} \mathrm{C}\right)$. Throughout the study, niosomal gel formulations were stored in aluminum-foil-sealed glass vials. The samples were withdrawn at different time intervals over a period of 1 month and drug leakage from the formulations was analyzed for drug content by using a UV spectrophotometer. ${ }^{26}$

\section{In vitro permeation study}

Permeation study of the prepared antiacne niosomal gel In vitro skin permeation studies were performed using vertical Franz diffusion cells (Rama Scientific, New Delhi, India) with an effective diffusion area of $2.54 \mathrm{~cm}^{2}$. The study was conducted using shaved Wistar rat skin. The skin was mounted on the receptor compartment with the stratum corneum side facing upwards into the donor compartment. The donor compartment was filled with $200 \mathrm{mg}$ of the antiacne niosomal gel containing $0.020 \%$ tretinoin and $0.600 \%$ BPO. A 25-mL aliquot of 1:1 (ethanol/ methanol:saline) $\mathrm{v} / \mathrm{v}$ was used as a receptor medium to maintain a sink condition. The receptor compartment was maintained at $37^{\circ} \mathrm{C}$ and stirred by a magnetic bar at $600 \mathrm{rpm}$. At appropriate time intervals, 3-mL aliquots of the receptor medium were withdrawn and immediately replaced by an equal volume of fresh receptor solution up to 24 hours. The samples were analyzed by a UV spectrophotometer at $234.8 \mathrm{~nm}$ for BPO and $348.6 \mathrm{~nm}$ for tretinoin. The flux was calculated for each component from the niosomal gel formulation using Wistar rat skin.

\section{Permeation study of the prepared antiacne cream}

The donor compartment was filled with $200 \mathrm{mg}$ of antiacne cream containing $0.020 \%$ tretinoin and $0.600 \%$ BPO. A $25-\mathrm{mL}$ aliquot of 1:1 (ethanol/methanol:saline) v/v was used as the receptor medium to maintain a sink condition. The receptor compartment was maintained at $37^{\circ} \mathrm{C}$ and stirred by a magnetic bar at $600 \mathrm{rpm}$. At appropriate time intervals, 3-mL aliquots of the receptor medium were withdrawn and immediately replaced by an equal volume of fresh receptor solution up to 24 hours. The samples were analyzed by a UV spectrophotometer at $234.8 \mathrm{~nm}$ for BPO and $348.6 \mathrm{~nm}$ for tretinoin. The release rate flux was calculated for each component from the cream formulation using Wistar rat skin.

\section{Permeation study of the prepared alcoholic solution}

The donor compartment was filled with $200 \mu \mathrm{L}$ of the antiacne alcoholic solution containing $0.020 \%$ tretinoin and $0.600 \%$ BPO. A 25-mL aliquot of 1:1 (ethanol/methanol:saline) $\mathrm{v} / \mathrm{v}$ was used as the receptor medium to maintain a sink condition. The receptor compartment was maintained at $37^{\circ} \mathrm{C}$ and stirred by a magnetic bar at $600 \mathrm{rpm}$. At appropriate intervals, 3-mL aliquots of the receptor medium were withdrawn and immediately replaced by an equal volume of fresh receptor solution for up to 24 hours. The samples were analyzed by a UV spectrophotometer at $234.8 \mathrm{~nm}$ for BPO and $348.6 \mathrm{~nm}$ for tretinoin. The flux was calculated for each component from the niosomal gel formulation using Wistar rat skin.

\section{In vitro skin-retention study}

The ability of vesicles to help retain the drug within the skin milieu (ie, the depot effect) was investigated by determining the amount of drug retained in the skin samples employed in permeation studies. After completion of the permeation experiment, skin mounted on the diffusion cell was removed. The skin was cleaned with cotton, dipped in saline solution, and blotted with tissue paper to remove any adhering formulation. Subsequently, the skin sample was homogenized with $20 \mathrm{~mL}$ of a chloroform:methanol/ethanol mixture $(2: 1, \mathrm{v} / \mathrm{v})$, for the extraction of a homogenate suspension which was thus obtained 
using filter paper. For determining the amount of drugs retained in skin milieu, skin was subjected to homogenization using chloroform:methanol/ethanol mixture $(2: 1, \mathrm{v} / \mathrm{v})$ for extraction of retained drugs, extracted drugs in chloroform:methanol/ethanol mixture $(2: 1, \mathrm{v} / \mathrm{v})$ after homogenization was filtered using filter paper. The drug content was quantified using a UV spectrophotometer at respective absorption maxima for BPO and TRA. ${ }^{27}$

\section{In vivo study}

The rabbit ear model was used to study comedone formation in order to assess the comedogenicity of cosmetics, toiletries, and dermatological preparation and to evaluate the potential of antiacne drugs. This comedo induction took place after about 2 weeks of repeated topical application of a chemical comedone such as $50 \%$ oleic acid. One set of rabbits was treated as a control and received no treatment, while the remaining two set of rabbits received treatment with $50 \%$ oleic acid and dimethyl sulfoxide for up to 28 days on the ventral aspect of the pinnas once a day. The total number of animals used was nine. A group of three animals were used in each of the three groups; one group was named as the control group, and the two other groups were used in the study. One of the test groups was treated with a niosomal gel and the other test groups was treated with cream. During the study, all animals were subjected to histological examination for assuring the effectiveness of the tested formulation.

\section{Stability of final niosomal gel}

Testing of the stability of the niosomal gel was performed in triplicate. The niosomal suspension was prepared separately for both the drugs and was dispersed in the gel. Three batches were prepared and studied at three temperature conditions (room temperature, refrigerated temperature, and $45^{\circ} \mathrm{C}$ ) to evaluate the impact of storage temperature on the stability of niosomes dispersed in the gel.

Stability studies were performed by considering a worst-case condition for the formulation in terms of the maximum interaction of excipient by minimizing the concentration of drug-loaded niosomes. In the performed study, a lower quantity of drug-loaded niosomes were dispersed in the gel and kept under the mentioned storage temperature condition to evaluate the combined effect of temperature and excipient over the niosomal gel.

The stability of the final formulation was determined by placing the formulation in a stable condition at room temperature, refrigerated temperature, and $45^{\circ} \mathrm{C}$ for 1 month, and the content of the added drug in the formulation was measured at various time intervals (1, 3, 7, 14, and 30 days); changes in the content of the final formulation were determined using a UV spectrophotometer (Systronic 2201).

\section{Results and discussion Incompatibility studies between drugs}

It was clear from infrared spectroscopy that the mixture of TRA and BPO was compatible because there were no alterations in band spectra when compared with each separate spectra of the drug. Two sharp bands were observed for TRA $\left(1,685.87 \mathrm{~cm}^{-1}\right.$ for $\mathrm{C}=\mathrm{O}$ str and $2,937.68 \mathrm{~cm}^{-1}$ for $\mathrm{O}-\mathrm{H}$ str $)$ and two bands for $\mathrm{BPO}\left(1,759.4 \mathrm{~cm}^{-1} \mathrm{C}=\mathrm{O}\right.$ str ester, $1,226.7 \mathrm{~cm}^{-1}$, $\mathrm{C}-\mathrm{O}$ str) (these are infrared spectroscopy band representative for functional group present in drugs. In TRA $\mathrm{C}=\mathrm{O}$ and $\mathrm{O}-\mathrm{H}$ group and $\mathrm{BPO} \mathrm{C}=\mathrm{O}$ for ester group and $\mathrm{C}-\mathrm{O}$ is present) in the mixture of TRA and BPO (Figure 1).
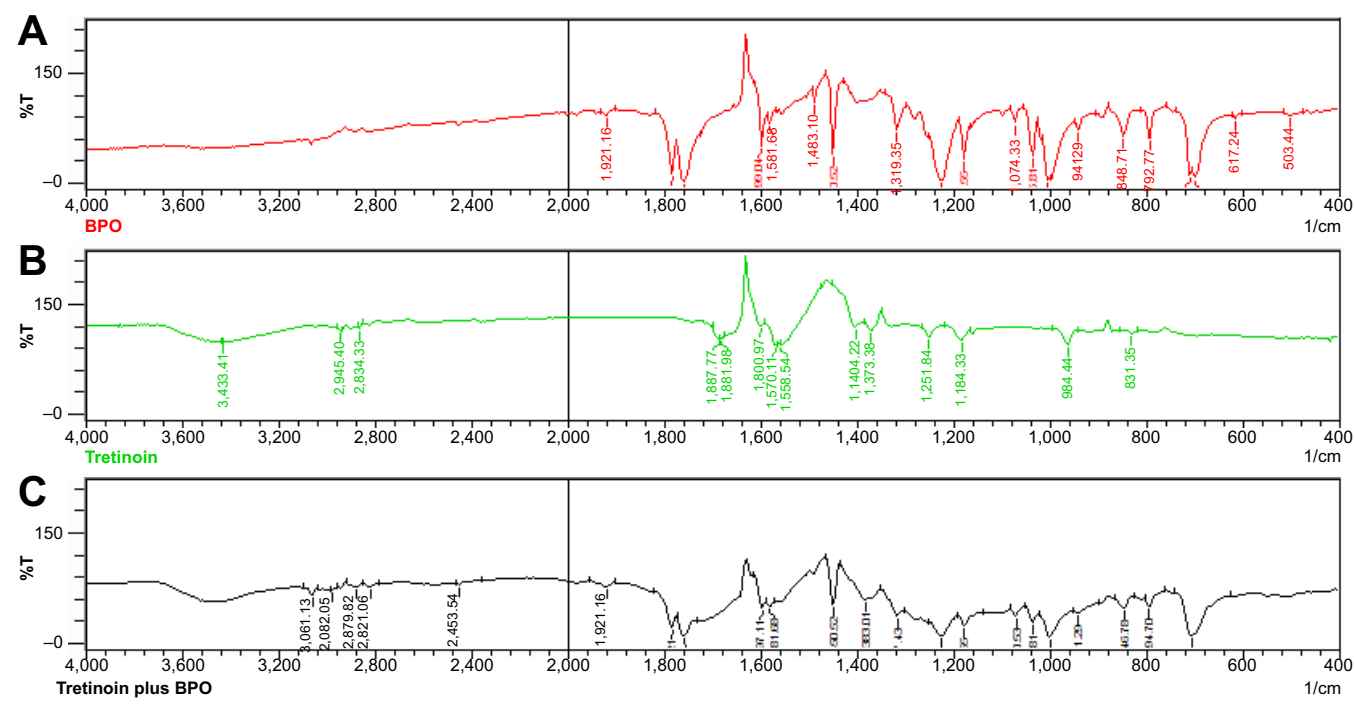

Figure I IR spectra of BPO, tretinoin, and the mixture of BPO and tretinoin.

Notes: (A) BPO; (B) tretinoin; (C) mixture of BPO and tretinoin.

Abbreviations: BPO, benzoyl peroxide; IR, infrared spectroscopy; $\mathrm{T}$, transmittance. 


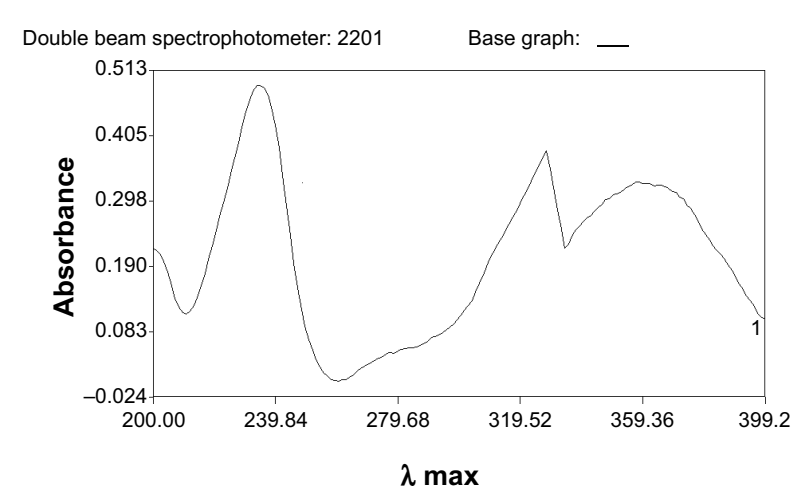

Figure 2 UV scan of the BPO and tretinoin mixture.

Abbreviations: BPO, benzoyl peroxide; max, maximum; UV, ultraviolet.

UV scan analysis also confirmed that the mixture of TRA and BPO were compatible because, upon scanning, the mixture of TRA and BPO $(10 \mu \mathrm{g} / \mathrm{mL})$ in the range of $200-$ to $400-\mathrm{nm}$ TRA gave absorption maxima at $348.6 \mathrm{~nm}$ and $234.8 \mathrm{~nm}$, respectively. The characteristic peak for TRA was obtained at $348.6 \mathrm{~nm}$ and for BPO it was obtained at $234.8 \mathrm{~nm}$. The interference of absorbance of one component with the absorbance of tretinoin at $234.8 \mathrm{~nm}$ was -0.059 and for BPO at $348.6 \mathrm{~nm}$ it was -0.026 . The results showed that there was no interference in the absorbance of each component in the mixture (Figure 2).

\section{Susceptibility testing of BPO against}

\section{S. epidermidis}

In this study, BPO was examined for antibacterial activity against $S$. epidermidis. The susceptibility study results (Table 3; Figure 3) showed that BPO could effectively inhibit the growth of $S$. epidermidis.

\section{Minimum inhibitory concentration of BPO for S. epidermidis}

The evaluated data demonstrated that BPO is effective against $S$. epidermidis at $28 \mu \mathrm{g} / \mathrm{mL}$, which shows that
$28 \mu \mathrm{g}$ is the minimum concentration of BPO that will be effective in killing $S$. epidermidis at the affected/pathogenic site (Figure 4).

\section{Characterization of niosomes Morphology analysis by transmission electron microscopy}

Transmission electron microscopy (TEM) was performed to determine vesicle formation and morphology. It was clear from the TEM analysis that uniform spherical niosomes were formed (Figures 5 and 6) and niosome photographs were taken from a photomicroscope at a $100 \times$ magnification (Figures 7 and 8) confirming the same. TEM micrographs showing the average size of niosomes without drugs demonstrated sizes in the range of $200-250 \mathrm{~nm}$.

\section{Particle size analysis by photon correlation spectroscopy (DLLS)}

DLLS analyses showed that some of the samples were polydispersed (polydispersivity index $=0.60$ ) and the reproducibility of vesicle sizes appeared to be good. The mean diameter of tretinoin-saturated and BPO-saturated vesicles was $616 \mathrm{~nm}$. In DLLS analysis, drug-containing noisomes, dispersed in gel then it was analyzed for estimating the size distribution through out the gel, which was showing that 616 $\mathrm{nm}$ average diameter of noisome present in the niosomal gel (Figure 9).

\section{Zeta potential}

The niosomal formulation containing drug-loaded niosomes (which was subjected to zeta potential analysis) had a zeta potential value of $-43 \mathrm{mV}$, which is a measure of net charge of the niosomes (Figure 10). This higher charge on the surface of vesicles produced a repulsive force between the vesicles which

Table 3 Zone of inhibition data

\begin{tabular}{llll}
\hline Serial number & Concentration $\mu \mathbf{g} / \mathbf{m L}$ & Average of zone of inhibition $\mathbf{( m m})$ & Standard deviation \\
\hline 1 & 50 & 12.5 & \pm 0.057 \\
2 & 100 & 12.5 & \pm 0.057 \\
3 & 150 & 13.9 & \pm 0.1 \\
4 & 200 & 14.9 & \pm 0.115 \\
5 & 250 & 14.8 & \pm 0.152 \\
6 & 300 & 14.9 & \pm 0.1 \\
7 & 350 & 14.9 & \pm 0.1 \\
8 & 400 & 15.5 & \pm 0.1 \\
9 & 450 & 15.5 & \pm 0.2 \\
10 & 500 & 17.0 & \pm 0.057 \\
11 & 1,000 & 16.9 & \pm 0.152 \\
12 & 1,500 & 17.0 & \pm 0.152 \\
13 & 2,000 & 16.9 & \pm 0.1 \\
\hline
\end{tabular}




\section{Zone of inhibition of Staphylococcus epidermidis using}

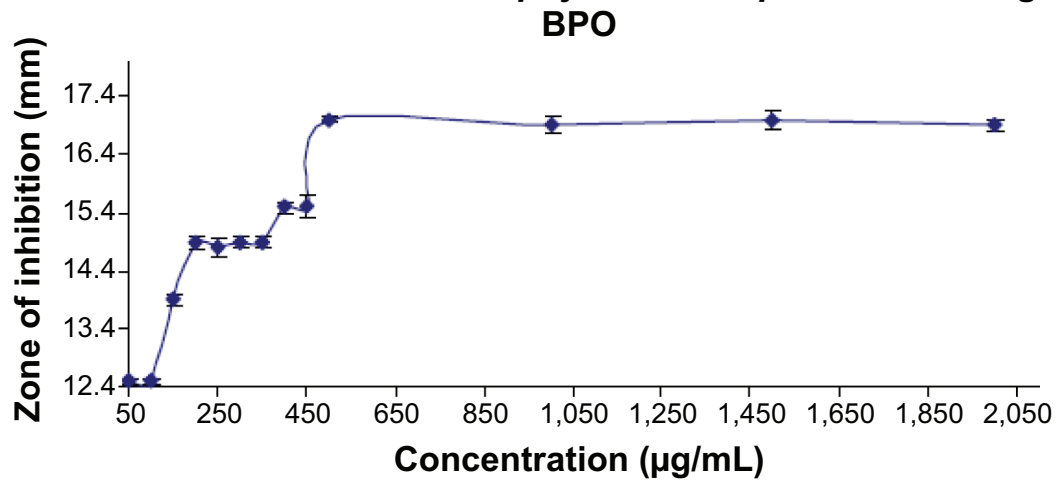

Figure 3 Susceptibility testing graph: BPO concentration versus zone of inhibition. Abbreviation: $\mathrm{BPO}$, benzoyl peroxide.

made them stable and devoid of agglomeration and faster settling, providing an evenly distributed suspension. From this, it can be concluded that the present niosomal formulations show good stability. In the present study, both drugs were loaded in the niosomes vesicles separately and then further dispersed in the gel formulation for application onto the skin. Drug loading will have an impact on zeta potential because the zeta potential indicates the degree of repulsion between adjacent, similarly charged particles in dispersion. Due to this consideration, separate loading of drugs in niosomes was completed, then processed subsequently for gel preparation.

\section{Encapsulation efficiency}

Under the same preparation condition, the encapsulation efficiency for both drugs was calculated. The encapsulation efficiency of BPO and tretinoin is given in Tables 1 and 2. Saline media for hydration was chosen for increasing the tretinoin encapsulation efficiency because the desired concentration of TRA required in niosomal gel formulation is less in comparison to BPO. The addition of a hypertonic salt solution to a suspension of niosomes brings about a reduction in diameter of niosomes, which leads to increased

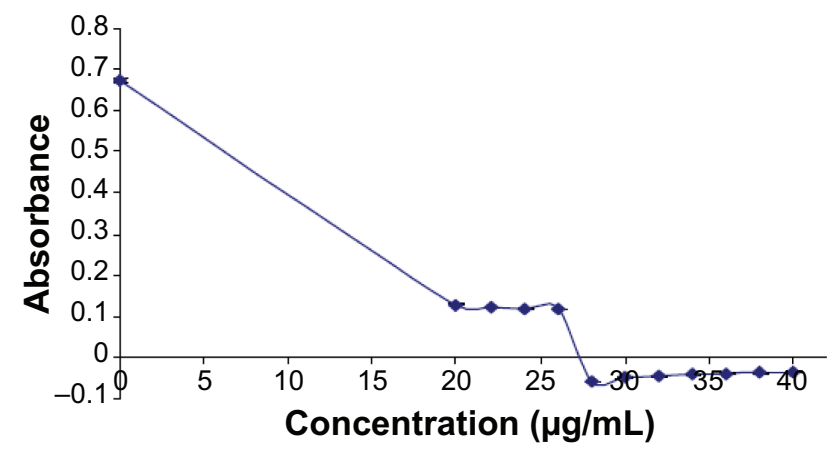

Figure 4 Minimum inhibitory concentration of benzoyl peroxide against Staphylococcus epidermidis. surface area and which proportionally leads to increased encapsulation efficiency.

DSC analysis for the determination of the gel-lipid transition temperature

DSC measurements were performed to determine the gel-lipid transition temperature or phase transformation of niosomes. With the increase of system temperature, the hydrocarbon chains in the ordered bilayer of vesicles were transformed from a rigid gel to facile liquid crystals meaning that the phase transition of the bilayer took place, indicating that the niosome transitioned to a liquid crystal. The transition of the niosomal formulation from a gel state (ordered state) to a liquid crystalline state (disordered state)

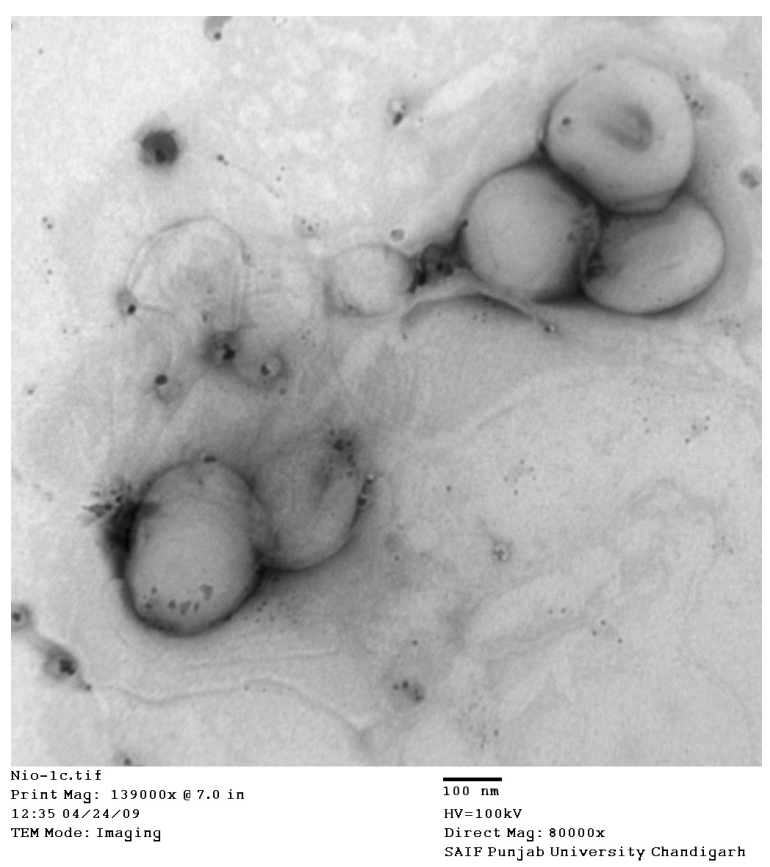

Figure $\mathbf{5}$ Transmission electron microscopy photograph of niosomes (negative staining). 


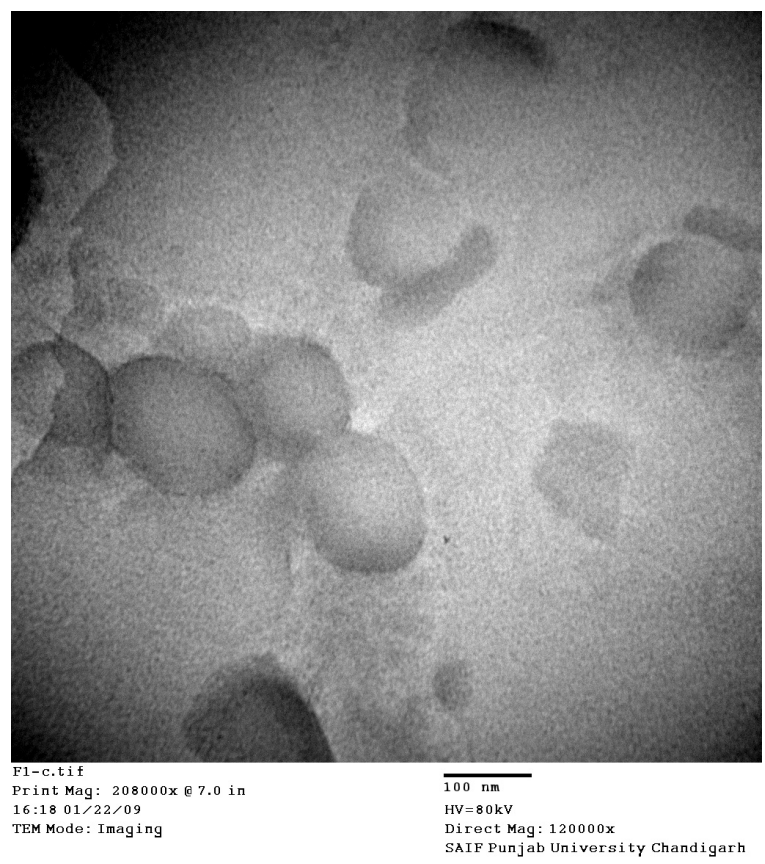

Figure 6 Transmission electron microscopy photograph of niosomes.

occurred at $56^{\circ} \mathrm{C}-58^{\circ} \mathrm{C}$ (Figure 11); this temperature range is, thus, the gel-lipid transition temperature of niosomes formed from span 60:cholesterol these ratio of Span 60: cholesterol (207:52 and 138:52 mg) used for preparation of TRA and BPO drug loaded niosomes respectively.

\section{Stability studies of the niosomal formulation}

Results showed that the niosomal gel formulation was quite stable at refrigeration and room temperatures since limited leakage of the drug was found at these temperatures. The drug retained at $45^{\circ} \mathrm{C}$ might have decreased due to the melting of the surfactant and lipid present in the formulation (Figures 12 and 13). Therefore, the niosomal gel formulations can be stored at either refrigeration or room temperature.

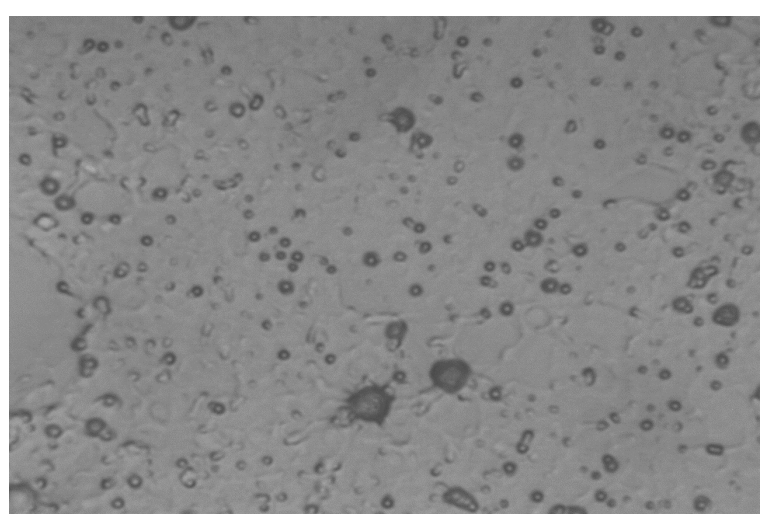

Figure 7 Photograph of benzoyl peroxide drug niosomes using a photomicroscope: $100 \times$ magnification.

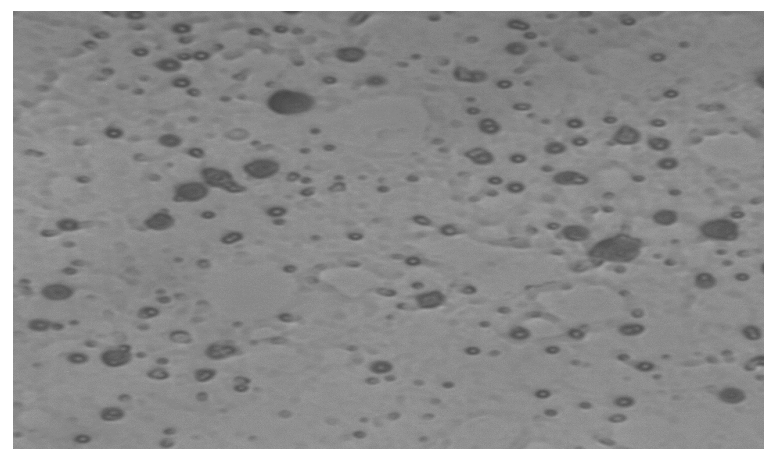

Figure 8 Photograph of tretinoin drug niosomes using a photomicroscope: 100x magnification.

\section{In vitro permeation study}

In vitro permeation study of niosomal gel

The mean amount of tretinoin and BPO permeated per unit of surface area was determined during 24-hour experiments. Figure 14 shows the permeation profiles of the niosomal gel (cumulative amounts of tretinoin and BPO permeated versus time). The permeated amount of tretinoin from the niosomal gel after 24 hours was calculated as $6.25 \pm 0.14 \mu \mathrm{g} / \mathrm{cm}^{2}$, and $5.04 \pm 0.014 \mu \mathrm{g} / \mathrm{cm}^{2}$ was calculated as the permeated amount of BPO from the niosomal gel after 24 hours.

\section{Permeation study of the prepared antiacne cream}

Figure 14 also shows the permeation profiles (the cumulative amounts of tretinoin and BPO permeated versus time) through the skin obtained from tretinoin and BPO containing $\mathrm{o} / \mathrm{w}$ cream. As can be seen in Figure 12, the permeation curves do not show a classic profile with a steady-state phase. The maximum permeated amount of tretinoin from the cream after 8 hours was calculated as $6.60 \pm 0.13 \mu \mathrm{g} / \mathrm{cm}^{2}$, and $7.91 \pm 0.023 \mu \mathrm{g} / \mathrm{cm}^{2}$ was the calculated permeated amount of BPO from the cream after 24 hours. Eight hours for tretinoin and 24 hours for BPO was considered the time taken for maximum amount permeated through skin using cream formulation. Cream is the w/o system; containing a major proportion of oil phase and due to the lipophilic nature of skin, the permeation content from cream is faster, which is the reason for faster permeation of tretinoin within 8 hours.

\section{Permeation study of the prepared alcoholic solution}

Figure 14 also shows the permeation profiles (the cumulative amounts of tretinoin and BPO permeated versus time) through the skin obtained from tretinoin and a BPO alcoholic solution. The maximum permeated amount of tretinoin from the alcoholic solution after 6 hours was calculated as $7.72 \pm 0.16 \mu \mathrm{g} / \mathrm{cm}^{2}$, and $12.18 \pm 0.013 \mu \mathrm{g} / \mathrm{cm}^{2}$ was the 


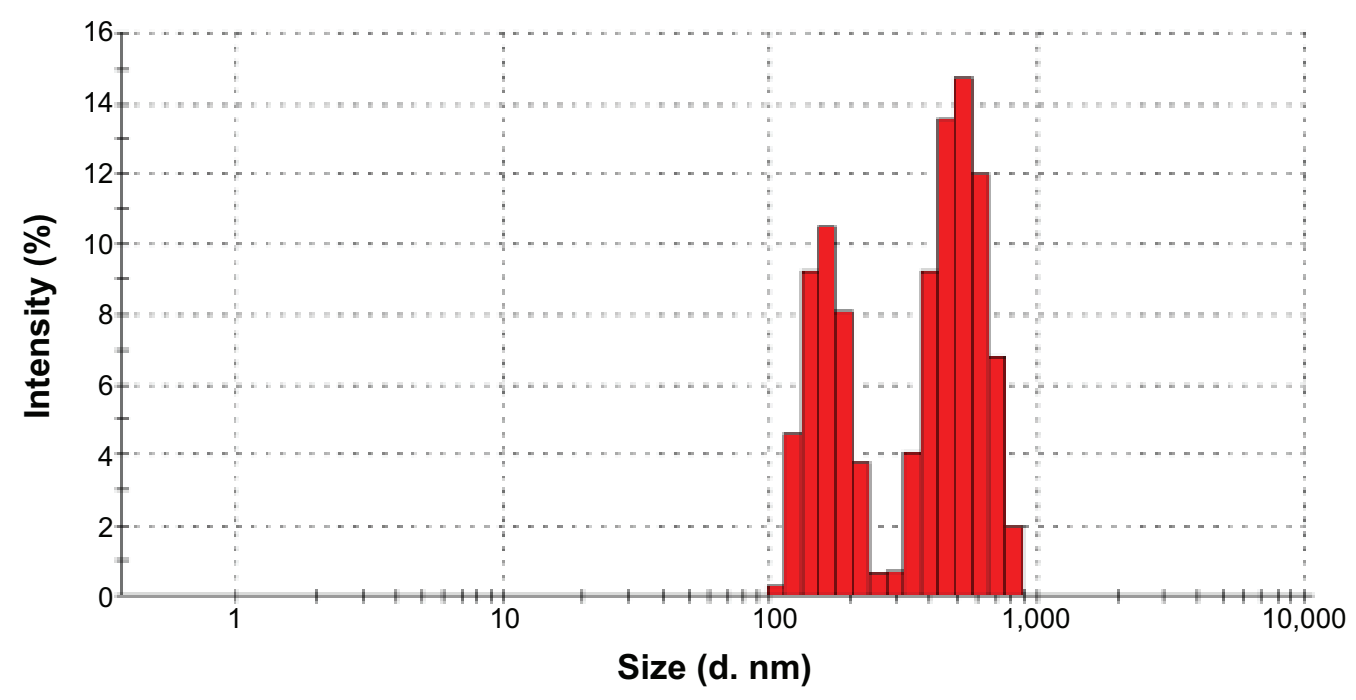

Figure 9 Statistical bar graph of particle size distribution in niosomal suspensions.

calculated permeated amount of BPO from the alcoholic solution after 6 hours.

\section{In vitro skin-retention study}

Results also showed that the drug content retained in the layers of skin was $11.54 \mu \mathrm{g}$ of tretinoin and $68.85 \mu \mathrm{g}$ of BPO from the cream, $2.68 \mu \mathrm{g}$ of tretinoin and $59.98 \mu \mathrm{g}$ of BPO from the alcoholic solution, and $15.54 \mu \mathrm{g}$ of tretinoin and $143.78 \mu \mathrm{g}$ of BPO from the niosomal gel. The drug retention was more in case of niosomal gel than the cream and alcoholic solution, so it can be concluded here for the first time that the gel was more effective than the cream and alcoholic solution (Figure 15).

\section{In vivo studies}

Results obtained from the histological studies showed that the prepared niosomal formulation was effective in the treatment of acne. Comparison of the control sample and treated pinna showed that there was a marked increase in volume of the sebaceous gland and several units of comedones present in the treated pinna. Moreover, for the acne-induced pinna treated with the prepared niosomal gel

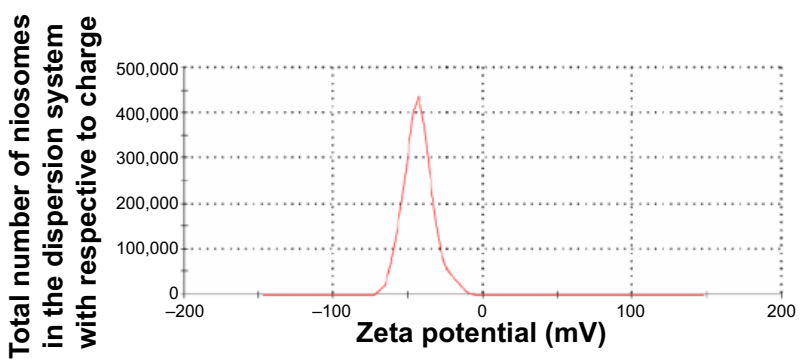

Figure 10 Zeta potential of niosomal suspensions. for up to 14 days, histological reports showed that there was a marked reduction in the volume of the sebaceous gland and no dilatation was present in the follicle (Figure 16). Similar results were obtained with the cream which contains $2.5 \%$ of BPO and $0.025 \%$ of tretinoin. From this comparative study, it can be concluded that the niosomal gel was more efficacious than the cream. It showed a similar therapeutic action when using a 4.16-fold lower dose of BPO. Moreover, histopathology micrograph showed that no comedones were present in the treated pinnas after treatment for 14 days with both antiacne cream and the niosomal formulation.

\section{Conclusion}

In summary, it is evident from the aforementioned study that niosomes showed better therapeutic activity than conventional dosage forms using formulations through the same route of administration. The greatest challenge with topical drug delivery is the barrier nature of skin, which restricts

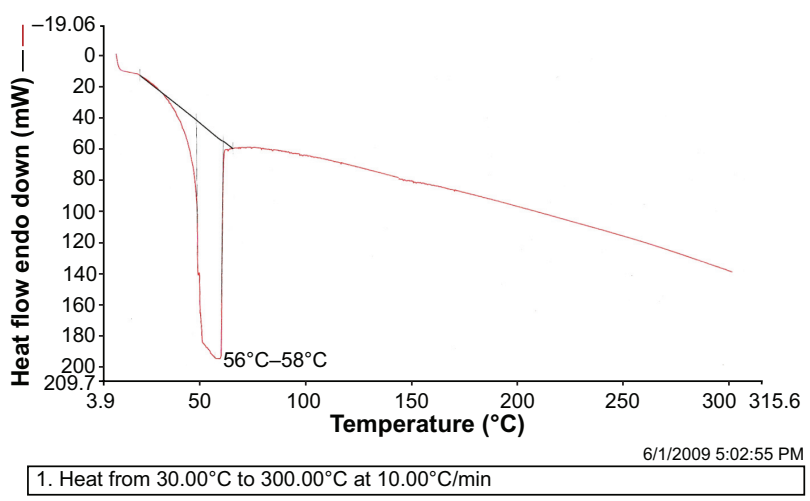

Figure I I Differential scanning calorimetry thermogram of the niosomal preparation. 


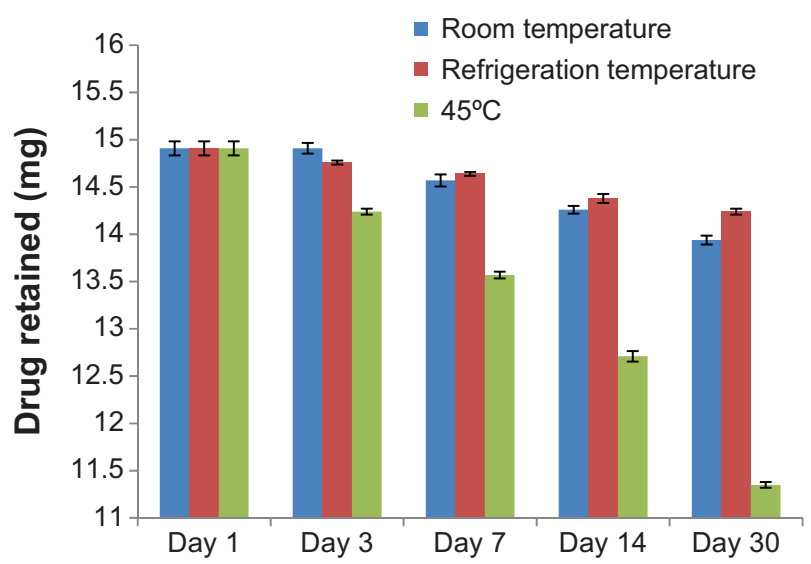

Figure 12 Drug (benzoyl peroxide) retained in niosomes at various temperature conditions for 30 days.

the entry of most drugs. Here, the present data proved that niosomes acted as the best vesicles in dermal drug delivery due to its nanometer size and their elastic nature. They acted as a drug carrier to deliver entrapped drug molecules into or across the skin and, owing to the individual lipid components, enhanced penetration into the stratum corneum and, subsequently, the alteration of the intercellular lipid lamellae within this skin layer. In vivo experiments demonstrated an interesting correlation between the better permeation capabilities of niosomes in comparison to other conventional dosage forms in terms of a better therapeutic efficacy at the affected site at lower doses of drugs present in the niosomal gel formulation. Comparative in vivo studies of the niosomal gel and antiacne cream of TRA and BPO showed that the niosomal gel was more efficacious than the antiacne cream

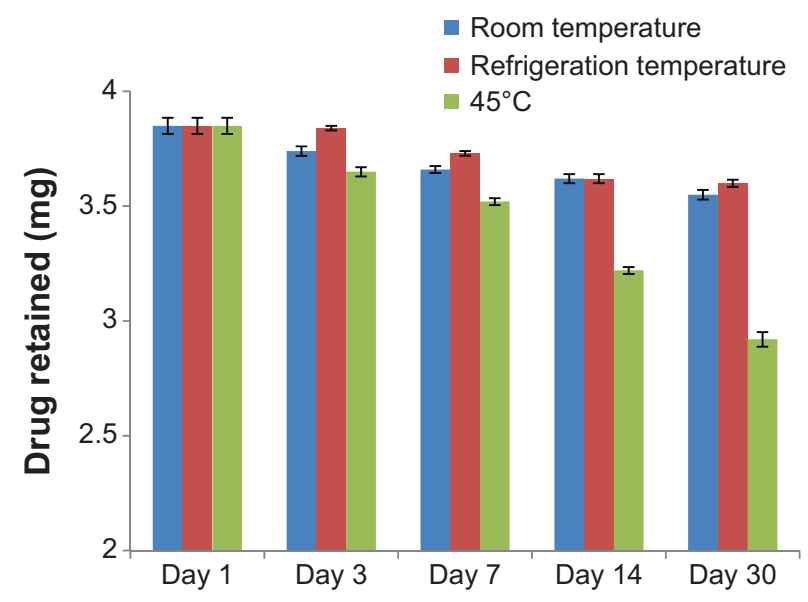

Figure 13 Drug (tretinoin) retained in niosomes at various temperature conditions for 30 days.

because niosomal gels increased the therapeutic index of a drug leading to a 4.16-fold reduction in dose of BPO in comparison to the antiacne cream. Antimicrobial susceptibility and MIC data showed that BPO has potent antibacterial action against acne-causing bacterium such as $S$. epidermidis at $28 \mu \mathrm{g} / \mathrm{mL}$. An ex vivo skin-retention study showed that the niosomal gel had maximum skin retention of BPO and TRA at the affected site. Due to maximum retention at the skin, acne-causing bacterium will not propagate, and the niosomal gel can maintain the MIC at the target site for prolonged periods of time due to a niosomal "depot mechanism". Based on the above data, it can be concluded that the nano vesicle (ie, niosomes)-based dosage forms developed here would have a better therapeutic efficacy at a lower dose in comparison to conventional dosage forms.
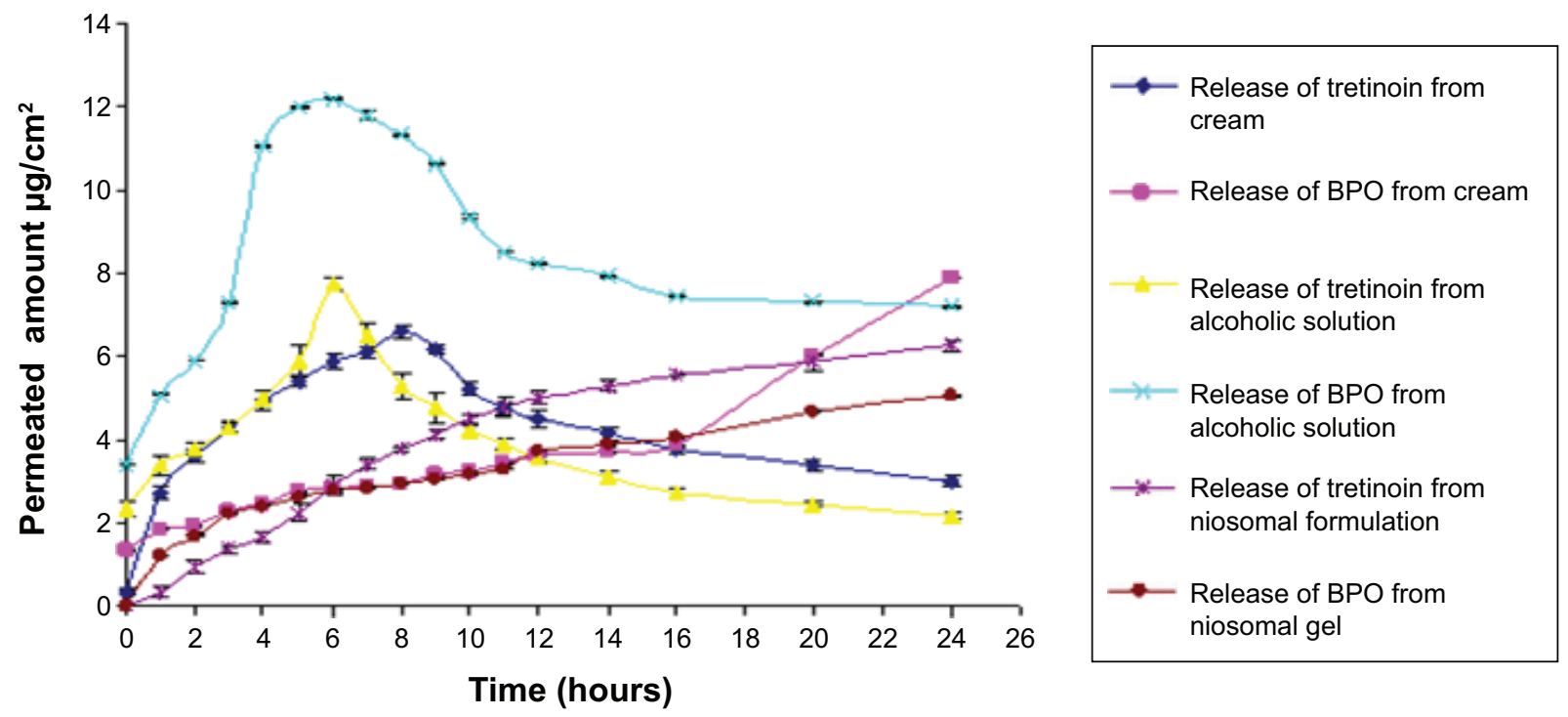

Figure 14 Permeation profile of BPO and tretinoin from cream, alcoholic solution, and niosomal gel. Abbreviation: BPO, benzoyl peroxide. 


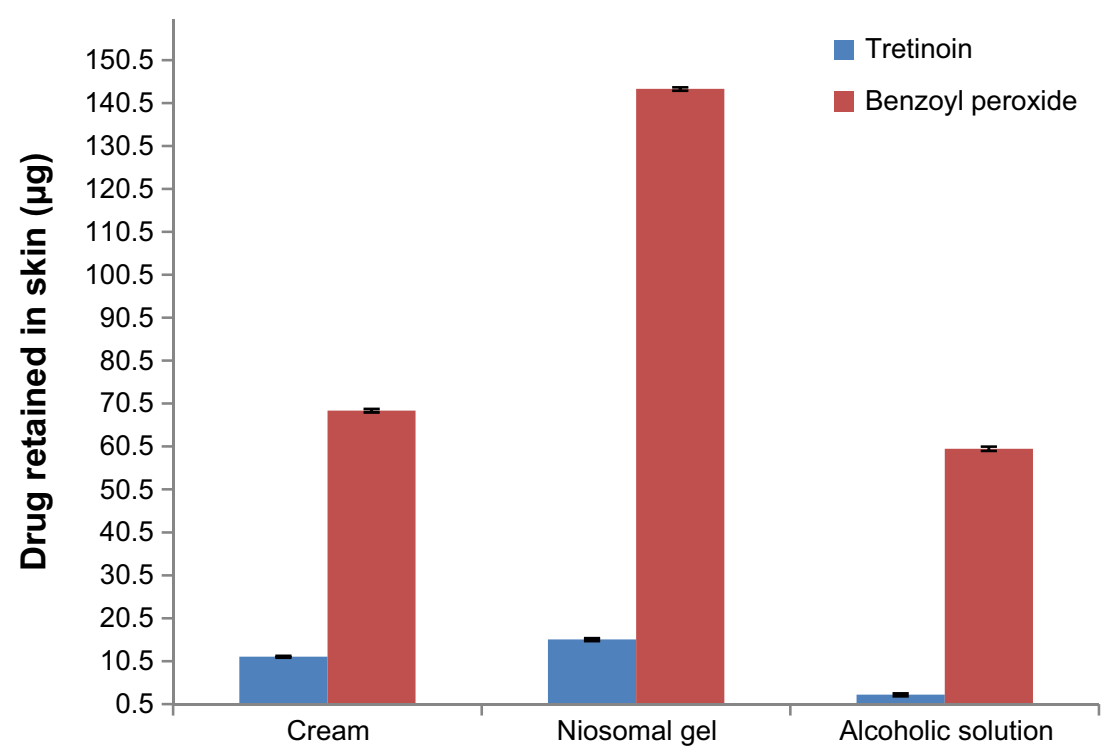

Figure 15 Comparison of the in vitro retention study of antiacne cream, alcoholic solution, and niosomal gel.

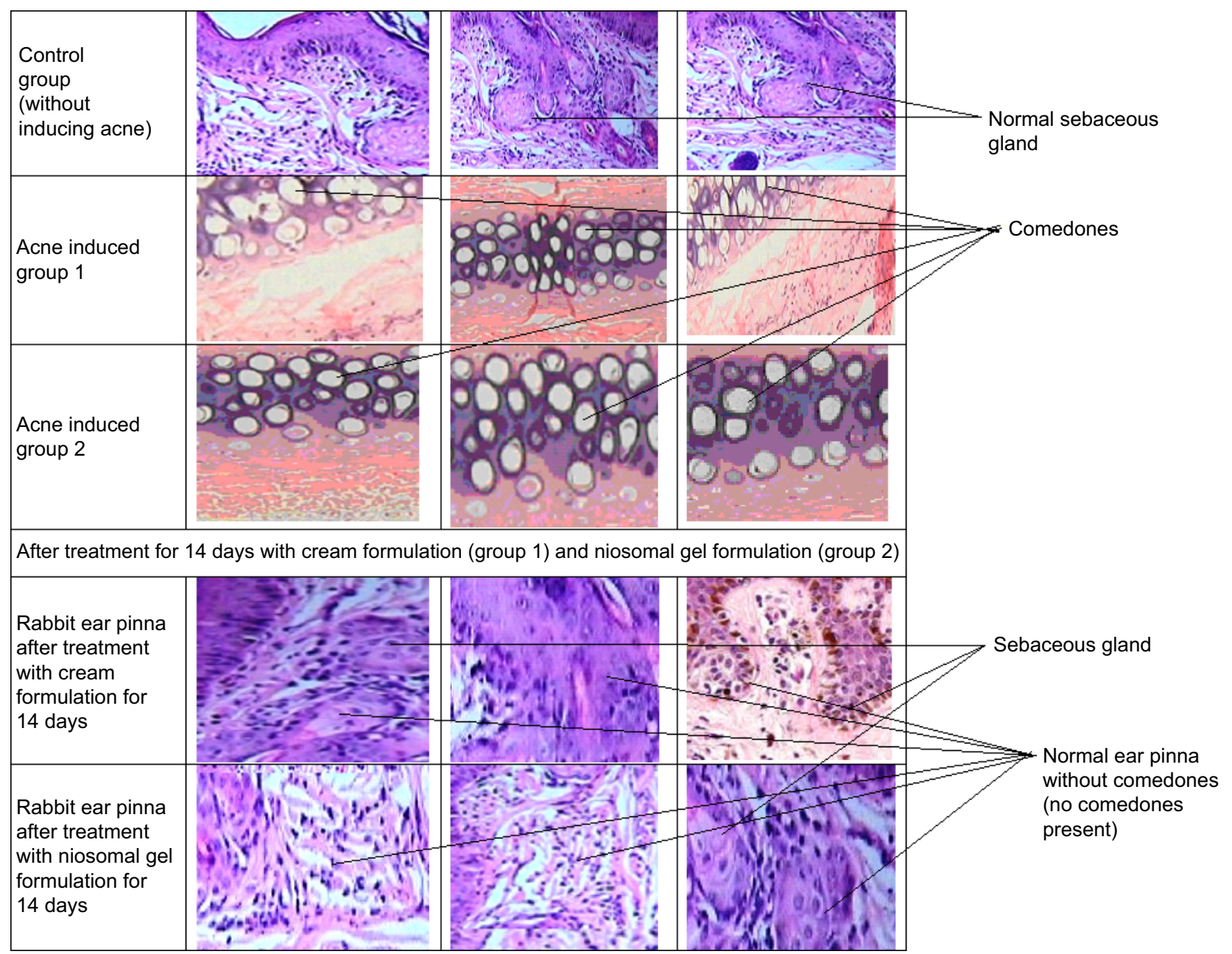

Figure 16 Comparative histopathological examination of control versus acne-induced pinna after treatment with cream for 14 days and after treatment with niosomal gel. 


\section{Acknowledgments}

The authors acknowledge technical support from Religare, SRL Daignostic SRL Ltd, Gurgoan, and financial support from the Lovely Professional University. The authors are grateful to the SAIF Team and Chandigarh for his technical assistance with the transmission electron microscopy analysis. Special thanks and gratitude to Shalaks Pharmaceutical, New Delhi, and HK Group, Mumbai for providing ex-gratis samples of TRA and BPO, respectively.

\section{Disclosure}

The authors report no conflicts of interest in this work.

\section{References}

1. Brown SK, Shalita AR. Acne vulgaris. Lancet. 1998;351:1871-1876.

2. Kumar GS, Jayaveera KN, Kumar A, Umachigi P, Vrushabendra BMS, Kumar DVK. Antimicrobial effects of Indian medicinal plants against acne-inducing bacteria. Tropical Journal of Pharmaceutical Research. 2007;6(2):717-723.

3. Shalita AR, Lee WL. Inflammatory acne. Dermatologic Clinics. 1983; 1:361-364.

4. Gollnick HP, Krautheim A. Topical treatment in acne: current status and future aspects. Dermatology. 2003;206:29-36.

5. Zaenglein AL. Topical retinoids in the treatment of acne vulgaris. Semin Cutan Med Surg. 2008;27:177-182.

6. Tucker R, Walton S. The role of benzoyl peroxide in management of acne vulgaris. Pharm J. 2007;279:48-53.

7. Spellman MC, Pincus SH. Efficacy and safety of azelaic acid and glycolic acid combination therapy compared with tretinoin therapy for acne. Clin Ther. 1998;20:4:711-721.

8. Unkles SE, Gemmell CG. Effect of clindamycin, erythromycin, lincomycin, and tetracycline on growth and extracellular lipase production by propionibacteria in vitro. Antimicrob Agents Chemother. 1982;21(1): 39-43.

9. Toyoda M, Morohashi M. An overview of topical antibiotics for acne treatment. Dermatology. 1998;196:130-134.

10. Mills $\mathrm{OH}$, Klingman AM. Treatment of acne vulgaris with topically applied erythromycin and tretinoin. Acta Derm Venereol. 1978;58: 555-557.

11. Honeywell-Nguyen PL, Bouwstra JA. Vesicles as a tool for transdermal and dermal delivery. Drug Discov Today Technol. 2005;2(1): 67-74.A
12. Escobar-Chávez JJ, Díaz-Torres R, Rodríguez-Cruz IM, et al. Nanocarriers for transdermal drug delivery. Research and Reports in Transdermal Drug Delivery. 2012;1:3-17.

13. Hao Y, Zhao F, Li N, Yang Y, Li K. Studies on a high encapsulation of colchicine by a niosome system. Int J Pharm. 2002;244:73-80.

14. Fang JY, Yu SY, Wu PC, Huang YB, Tsai YH. In vitro skin permeation of estradiol from various proniosome formulations. Int J Pharm. 2001; 215:91-99.

15. Manosroi A, Wongtrakul P, Manosroi J, et al. The entrapment of kojic oleate in bilayer vesicles. Int J Pharm. 2005;298:13-25.

16. Vyas SP, Venkatesan N. Poly(phthaloyl-L-lysine)-coated multilamellar vesicles for controlled drug delivery: in vitro and in vivo performance evaluation. Pharm Acta Helv. 1999;74:51-58.

17. Ito M, Motoyoshi K, Suzuki M, Sato Y. Sebaceous gland hyperplasia on rabbit pinna induced by tetradecane. J Invest Dermatol. 1985;85: 249-254.

18. Vogel HG. Drug Discovery and Evaluation: Pharmacological Assay. In: Med, Gerhard SCH, editors. Berlin, Heidelberg: Springer Verlag; 2002:1339-1340.

19. Chomnawang MT, Surassmo S, Nukoolkarn VS, Gritsanapan W. Antimicrobial effects of Thai medicinal plants against acne-inducing bacteria. J Ethnopharmacol. 2005;101:330-333.

20. Agarwal R, Katare OP, Vyas SP. Preparation and in vitro evaluation of liposomal/niosomal delivery systems for antipsoriatic drug dithranol. Int J Pharm. 2001;228:43-52.

21. Arunothayanun P, Bernard MS, Craig DQM, Uchegbu IF, Florence AT. The effect of processing variables on the physical characteristics of non-ionic surfactant vesicles (niosomes) formed from a hexadecyl diglycerol ether. Int J Pharm. 2000;201:7-14.

22. Bhatia A, Kumar R, Katare OP. Tamoxifen in topical liposomes: development, characterization and in vitro evaluation. J Pharm Pharm Sci. 2004;7(2):252-259.

23. Gupta A, Prajapati SK, Balamurugan M, Singh M, Bhatia D. Design and development of a proniosomal transdermal drug delivery system for captopril. Tropical Journal of Pharmaceutical Research. 2007;6(2): 687-693.

24. Hathout RM, Mansour S, Mortada ND, Guinedi AS. Liposomes as an ocular delivery system for acetazolamide: in vitro and in vivo studies. AAPS Pharm Sci Tech. 2007;8(1):1.

25. Li LC, Tian Y. Zeta potential. In: Swarbick J, Boylan JC. Encyclopedia of pharmaceutical technology. Vol 6. 3rd edition. 1995:4117-4128.

26. Hua W, Liu T. Preparation and properties of highly stable innocuous niosome in Span 80/PEG 400/H $\mathrm{H}_{2} \mathrm{O}$ system. Colloids Surf A Physicochem Eng Asp. 2007;302:377-382.

27. Manconi M, Sinico C, Valenti D, Lai F, Fadda AM. Niosomes as carriers for tretinoin. III. A study into the in vitro cutaneous delivery of vesicle-incorporated tretinoin. Int J Pharm. 2006;311:11-19.
International Journal of Nanomedicine

\section{Publish your work in this journal}

The International Journal of Nanomedicine is an international, peerreviewed journal focusing on the application of nanotechnology in diagnostics, therapeutics, and drug delivery systems throughout the biomedical field. This journal is indexed on PubMed Central, MedLine, CAS, SciSearch ${ }^{\circledR}$, Current Contents ${ }^{\circledR} /$ Clinical Medicine,
Dovepress

Journal Citation Reports/Science Edition, EMBase, Scopus and the Elsevier Bibliographic databases. The manuscript management system is completely online and includes a very quick and fair peer-review system, which is all easy to use. Visit http://www.dovepress.com/ testimonials.php to read real quotes from published authors. 